\title{
Structural characterization of high-performance catalysts for partial oxidation- the high-resolution and analytical electron microscopy approach
}

\author{
Jakob B. Wagner ${ }^{\mathrm{a}}$, Dang Sheng Su${ }^{\mathrm{a}}$, Stephan A. Schunk ${ }^{\mathrm{b}}$, Hartmut Hibst ${ }^{\mathrm{c}}$, Jochen Petzoldt ${ }^{\mathrm{c}}$ and \\ Robert Schlögl ${ }^{\mathrm{a}}$ \\ ${ }^{a}$ Abteilung Anorganische Chemie, Fritz-Haber-Institut der Max-Planck-Gesellschaft, Faradayweg 4- \\ 6, 14195, Berlin, Germany \\ ${ }^{\mathrm{b}}$ hte Aktiengesellschaft, Kurpfalzring 104, 69123, Heidelberg, Germany \\ ${ }^{\mathrm{c}} \mathrm{GCC} / \mathrm{S}$, BASF Aktiengesellschaft, 67056, Ludwigshafen, Germany
}

\begin{abstract}
A set of Mo, V, and $\mathrm{W}$ mixed-oxide samples with additives $\mathrm{P}$ and $\mathrm{Cs}$ showing high performance in selective partial oxidation of propene is studied by means of elemental mapping and crystal structure determination using scanning and transmission electron microscopy, energy-dispersive X-ray spectroscopy (EDS), and electron energy loss spectroscopy (EELS) as supplement to XRD measurements which are insufficient for phase determination. The set is divided into two subsets of samples denoted A and B, where subset A samples show the highest performance in selective partial oxidation of propene. These high-performance catalysts are all predominated by structures of the $\mathrm{Mo}_{5} \mathrm{O}_{14}$ type in contrast to a negligible amount of the orthorhombic $\mathrm{MoO}_{3}$, which is known to favor total oxidation.
\end{abstract}

Keywords: Molybdenum oxide; Partial oxidation; Structure determination; HREM; EELS; EDS 


\section{Introduction}

The industrial and economic relevance of research in selective partial oxidation catalysts is clear as about one-quarter of all organic products produced worldwide are synthesized via catalytic selective partial oxidation reactions [1]. These industrial processes are in general highly developed, but might be improved through high-throughput design and testing. However, more fundamental insight and understanding about the catalytically active structures and their relations to the catalytic performance are necessary for improving catalysts for such industrial processes in an efficient way.

The Mars-van Krevelen mechanism is widely accepted for selective partial oxidation reactions. The mechanism describes the role of the so-called active "lattice" oxygen for selective partial oxidation [2]. Coordinately unsaturated Mo atoms are suggested to act as adsorption sites for the alkenes under formation of an allylic intermediate [3 and 4]. Oxygen insertion occurs via a $\sigma$ bond formation between the lattice oxygen and the allylic intermediate. The oxygenated product desorbs and leaves an oxygen vacancy at the catalyst surface [5]. This vacancy is reoxidized via oxygen diffusion through either the near-surface layer or the bulk. The nature of the lattice oxygen is yet not fully unraveled and it is still under debate which of the possible metal-oxygen species actually take part in the oxygen insertion reaction [3 and 6], but it is clear that the diffusion kinetics are strongly affected by the elemental composition and geometric structures of the catalyst phases. Furthermore, the electronic properties of reduced molybdenum oxides, i.e., high electron density at the surface, may effect the oxygen activation.

Several MoVW-supported catalysts are currently used in industry for synthesis of acrylic acid [7, 8, 9, 10 and 11] and several publications deal with promoted MoVW oxide catalysts for acrolein oxidation [12, 13, 14 and 15]. These mixed Mo, V, and $\mathrm{W}$ oxide catalyst systems are rather unique in the sense that they reach high selectivities to acrylic acid of above $90 \%$ and simultaneously high acrolein conversions of also above $90 \%$. Furthermore, the high activity and selectivity of these catalysts remain constant for a long time of operation in the industrial process.

Previously, the $\mathrm{Mo}_{5} \mathrm{O}_{14}$ type phase is found to be highly important for selective partial oxidation catalysis, for example, methanol oxidation [16], acrolein oxidation [17], and propene oxidation [18]. Proceeding work [16, 17, 18 and 19] shows that the selectivity for partial oxidation products could be considerably augmented when the amount of the $\mathrm{Mo}_{5} \mathrm{O}_{14}$-type phase was increased at the expense of orthorhombic $\mathrm{MoO}_{3}$.

The structural dependence of the catalytic performance of $\mathrm{Mo}_{5} \mathrm{O}_{14}$-type structures and orthorhombic $\mathrm{MoO}_{3}$ in selective partial oxidation must be discussed in the scope of metal-oxide bond lengths [20]. In general, the shorter the metal-oxygen distance, the more basic the oxygen functionality. Basic, nucleophilic oxygen groups on the catalyst surface are necessary for C---H bond activation [6]. Weaker and longer metal-oxygen bonds favor the insertion of oxygen into organic molecules. 
Furthermore, selective oxidation catalysts have to discriminate, for example, between $\alpha-\mathrm{H}$ atoms of aldehydes and vinylic hydrogen atoms. Again the metal-oxygen bond strength plays the key role. Very strongly bound oxygens will unselectively activate C---H bonds, and very labile M---O groups will lead to total oxidation. Both types of oxygen bonds are present in $\mathrm{MoO}_{3-x}$ [20]. The optimization of the catalyst with respect to selectively $\mathrm{H}$-abstracting and oxygen insertion may be found in the optimization of the metal-oxygen bond length. Promoters, e.g., V, Nb, Ta, and $\mathrm{W}$, stabilize intermediate Mo oxides with proper metal-oxygen bond lengths such as $\mathrm{Mo}_{5} \mathrm{O}_{14}$. Furthermore, the catalyst must be optimized with respect to oxygen transferring functionalities according to the Marsvan Krevelen mechanism [21].

The motif of the $\mathrm{Mo}_{5} \mathrm{O}_{14}$-type structure consists of five corner-sharing octahedrally coordinated metal centers forming a pentagonal bipyramid [22]. In Fig. 1 the structure is shown along the [001] and the [100] zone axis. The $\mathrm{Mo}_{5} \mathrm{O}_{14}$-type structure can be described with unit cell parameters of $a=b=2.30 \mathrm{~nm}$ and $c=0.39 \mathrm{~nm}$. However, the exact values of the lattice parameters are dependent on the $\mathrm{V}$ and $\mathrm{W}$ content in the sample. The corner-sharing open structure makes it easy to imagine coordination sphere changes which might explain its activity in partial oxidation catalysis. Furthermore, stable $\mathrm{Mo}_{5} \mathrm{O}_{14^{-}}$ type binary molybdenum-based oxides doped with different elements such as $\mathrm{Nb}, \mathrm{W}$, and Ta have been synthesized at a wide temperature range and a broad variation of element ratios [23 and 24].

The oxygen-transferring functionalities at the atomic level are considered as motion of octahedral units reversibly changing their cross-linking. The activation energy and hence the reaction kinetics are optimized if the units can change the cross-linking independent of each other; i.e., the movements are decoupled in space and time. In this respect it is obvious that the $\mathrm{Mo}_{5} \mathrm{O}_{14}$-type structure is superior to conventional shear structures of metal oxides, such as $\mathrm{Mo}_{3} \mathrm{VO}_{11-x}$ and others, discussed in the literature [25, 26, 27, 28 and 29] as they require cooperative movements of chains of building blocks. The basic structure, of course, must be preserved during the oxygen transfer and phase separation and crystallization avoided. Such structural catalytic deactivating processes have not been observed in the present samples after catalytic reaction.

High-resolution and analytical TEM is a powerful technique for providing local information of specimens with respect to defects, peripheral structure, and composition in the form of lattice fringe images and local EELS measurements. This information is a significant supplement to the information acquired from averaging techniques such as XRD which are more sensitive to the bulk structure and do not provide sufficient information of the catalytically relevant surface structures in the material.

A series of papers have been devoted to the deeper understanding of the relation between catalyst structure and catalytic performance in MoVW oxide catalysts [16, 17, 19 and 21]. In the present work the study of the high-performance catalysts for selective partial oxidation reveals a majority of the 
$\mathrm{Mo}_{5} \mathrm{O}_{14}$-type structure, which is considered as the active phase in the molybdenum-based catalysts for selective partial oxidation of propene by the means of high-resolution and analytical TEM.

\section{Experimental}

The catalyst are prepared according to the following procedure: a transparent aqueous solution of ammonium tungstate, vanadate, and molybdate of the respective stoichiometry of the target solid is spray-dried at between 350 and $250{ }^{\circ} \mathrm{C}$ head temperature and 150 to $110^{\circ} \mathrm{C}$ offgas temperature. The resulting solid is kneaded with water and dried at $140{ }^{\circ} \mathrm{C}$ in a forced convection oven. Subsequently the samples are calcined in air for $2.5 \mathrm{~h}$ raising the temperature from 230 to $350{ }^{\circ} \mathrm{C}$ followed by increasing the temperature up to $400{ }^{\circ} \mathrm{C}$ within $2.5 \mathrm{~h}$ [for comparable materials and preparative procedures please see EP 0774 297, 1995, to BASF AG]. The materials show characteristic reflections in the X-ray diffractogram at $22.3^{\circ} 2 \theta$ and $48.8^{\circ} 2 \theta$. In addition, a typical broad halo over the range of 20 to $40^{\circ} 2 \theta$ can be observed (for reference diffractograms, see [16] and [19]). Surface areas determined by nitrogen sorption of the calcined materials will generally range between 2 and $4 \mathrm{~m}^{2} / \mathrm{g}$. The color of the calcined solid multicomponent oxide will generally be black, due to the presence of vanadium in a state of valence of + IV. For further treatment of the multicomponent oxides, cesium and phosphorus (as acetate and acid in aqueous solutions) are added as dopants to the calcined materials via two different impregnation techniques. For subset $\mathrm{A}$ an excess of liquid used for impregnation with regard to the total water uptake is employed, for subset B the amount of liquid with regard to the total water uptake is lower than the total water uptake. Both samples are allowed to settle with the impregnation solution at room temperature for $1 \mathrm{~h}$ and then dried at $80^{\circ} \mathrm{C}$ for $24 \mathrm{~h}$ in a forced convection oven, followed by thermal treatment at $400{ }^{\circ} \mathrm{C}$ under nitrogen. The final catalyst is employed as compacted and grinded granulate of a size of 300 to $500 \mu \mathrm{m}$.

The powder specimens are prepared for electron microscopy by standard preparation. The powders are crushed using a mortar and pestle and dispersed dry on meshed copper grids coated with a holey carbon film. The samples are studied in a Philips CM 200 FEG electron microscope equipped with a Gatan Image filter. Elemental information is obtained by EELS and EDS analysis, while crystallographic information is obtained from high-resolution images revealing lattice fringes and selected area electron diffraction (SAED) patterns. Special care must be taken by fast image acquisition to minimize the electron irradiation damage of the oxide specimens, which is one of the largest problems using this technique. Scanning electron microscopy (SEM) images are acquired on a $\mathrm{S} 4000$ FEG microscope (Hitachi). The acceleration voltage is set to $10 \mathrm{kV}$ and the working distance to $8 \mathrm{~mm}$.

Electron energy loss spectra of the catalyst samples are acquired in the energy loss interval 200-700 $\mathrm{eV}$ with an energy dispersion of $0.5 \mathrm{eV}$. With this setup the Mo $\mathrm{M}$ edges, the $\mathrm{V} \mathrm{L}_{2,3}$ edges, and the $\mathrm{O}$ $\mathrm{K}$ edge are present in the same spectra. The $\mathrm{W}$ M edges are located at approximately $1800 \mathrm{eV}$; hence, the signal to noise ratio is too low to detect the low concentration of $\mathrm{W}$ in the present samples. The 
energy loss near-edge structure (ELNES) of the $\mathrm{V} \mathrm{L}_{2,3}$ edges and the $\mathrm{O} \mathrm{K}$ edge are revealed in spectra acquired in the interval $470-570 \mathrm{eV}$ with an energy dispersion of $0.1 \mathrm{eV}$.

EDS analysis is performed on a Philips CM $200 \mathrm{LaB}_{6}$ electron microscope using a DX 4 analyzer system (EDAX) equipped with a Li-doped $\mathrm{Si}$ detector with a resolution of $140 \mathrm{eV}$ at $5.9 \mathrm{kV}$. Elemental maps of molybdenum, vanadium, and tungsten are acquired by EDS in STEM mode in order to study the elemental distribution throughout the agglomerates. The elemental maps are acquired cumulatively from 256 frames resulting in a total acquisition time of $\sim 1 \mathrm{~h}$.

\section{Results}

\subsection{Morphology (SEM/TEM)}

Fig. 2 shows characteristic SEM images of the samples and corresponding TEM images. The samples from subset $\mathrm{A}$ reveal a rough and porous surface as shown in Fig. 2a. In Fig. $2 b$ the surface of a sample from subset $B$ is shown. The surface is less porous and more smooth than the surface of subset A samples. The differences, in morphology are seen in the TEM images as well. In Fig. $2 \mathrm{c}$ the crystals of a subset A sample are sized 10-20 nm and clearly distinguishable. This primary structure is lost in the samples from subset B as the agglomerates become more close packed and the single crystallites are no longer distinguishable in TEM images such as Fig. 2d. Hence, the smaller crystal size and the higher porosity in the subset A samples lead to higher surface area to mass ratios which might additionally improve the catalytic performance compared to subset B samples, as discussed below.

\subsection{Microstructure (HRTEM/electron diffraction)}

The atomic structures are studied by high-resolution TEM (lattice fringe images) and electron diffraction. The crystalline structures of the samples can be divided into two groups. One group is extremely beam sensitive. After less than $1 \mathrm{~min}$ of irradiation, the crystalline phases become completely amorphous. In Fig. 3a the crystalline structure of such a highly beam sensitive phase is shown. The upper part of the image shows an amorphous area, which in Fig. $3 \mathrm{~b}$ has grown to fill out the whole image view after less than $1 \mathrm{~min}$ of additional low-dose electron beam irradiation. The perpendicular lattice fringe distances found in Fig. $3 \mathrm{a}$ are 0.79 and $0.55 \mathrm{~nm}$.

The second group of crystalline structures found in the samples is more stable under electron beam irradiation. No change in their crystalline structure is observed within 10-20 min. of exposure time. Fig. 4 shows a lattice fringe image of a crystal stable under irradiation. The resolution of the HRTEM image is limited due to unfavorable defocussing and misorientation of the crystal with respect to the incident electron beam, as seen in the fast Fourier transform (FFT) inset in Fig. 4. The distance between the spots in the cubic pattern found in the FFT corresponds to $2.25 \mathrm{~nm}$ in real space, which fits very well with the unit cell parameter $\left(d_{100}\right)$ of $\mathrm{Mo}_{5} \mathrm{O}_{14}$ in the [100] direction. Thus, the lattice 
fringe image shows a $\mathrm{Mo}_{5} \mathrm{O}_{14}$-type crystal structure slightly tilted from the [001] zone axis. The large unit cell parameter of the $\mathrm{Mo}_{5} \mathrm{O}_{14}$-type structure in the [100] is giving rise to a pseudolamellar nature when viewed along the [010] zone axis. In Fig. 5a such a pseudolamellar nature is shown. The FFT of the HRTEM image (Fig. 5b) reveals parallel streaks separated with a distance corresponding to $0.38 \mathrm{~nm}$ which coincide with the $d_{001}$ distance in the $\mathrm{Mo}_{5} \mathrm{O}_{14}$-type structure. The (one-dimensional) ordering observed in the crystal destroyed in the outermost 1-2 nm of the crystal, as the well-defined crystal is encapsulated by a 0.5 - to 2-nm thick structurally ill-defined layer. To avoid effects of the crystal edge in the FFT, the transformation is performed on an area excluding the outermost $3 \mathrm{~nm}$ of the crystal. However, as projected images are considered, ill-defined layers might disturb the imaging along the electron beam. The streaks observed in the FFTs and electron diffraction patterns (not shown here), which characterize the pseudolamellar nature of the structure, are observed with several different interspacings. Lattice fringe images of pseudolamellar structures ordered in two dimensions as expected in the $\mathrm{Mo}_{5} \mathrm{O}_{14}$-type structure viewed with misalignments to the [010] zone axis reveal a broad spectrum of interspacings corresponding to different plane spacings in the structure.

Both the very beam-sensitive crystalline structures and the more stable phases are found in all the studied material, both in subset A and subset B samples. The amount of the different crystalline phases is not estimated as HRTEM is not suitable for quantification of the crystalline structures. Here, it must be pointed out that XRD analysis is not well suited for detecting defective structures observed in the HRTEM images, in particular, in the particle periphery. In Fig. 6 an XRD pattern of the samples is shown. The main features in the patterns coincide for spectra acquired from subset $\mathrm{A}$ and subset $\mathrm{B}$ samples. However, the profile of the pattern does not give any unique phase determination. The typical XRD pattern is compared with measured spacings between lattice fringes in the high-resolution images of the samples. The broad spectrum of lattice spacings observed in the HRTEM images does not distinctively show up in the XRD powder spectrum. This indicates that XRD is an insufficient way to (quantitatively) characterize the present catalyst samples.

\subsection{EDS mapping}

Representative elemental maps of Mo, $\mathrm{V}$, and $\mathrm{W}$ of a subset $\mathrm{A}$ sample and a subset $\mathrm{B}$ sample are shown in Fig. 7 and Fig. 8, respectively. In general, the high Mo content shows as denser maps, as seen in Fig. 7 and Fig. 8. The relative ratio between the metals in the oxide is revealed by comparing the Mo, the V, and the $\mathrm{W}$ map. In Fig. 7 the metals are seen to be equally distributed in the agglomerate, as no variations are observed in the maps indicating the absence of phase separation. No variations in the elemental distribution are observed in the subset B samples either ( Fig. 8). However, the denser map in Fig. 8c compared to the map in Fig. 7c shows the higher vanadium content in the subset B agglomerates. 


\subsection{EELS measurements (ELNES)}

The short lifetime of the electron beam-sensitive phase does not allow reliable EELS acquirements impeding spatial-resolved analytical characterization of this phase. In the following paragraphs only the irradiation stable phase is considered. Fig. 9a shows representative spectra of subset A and subset B catalysts after background subtraction and normalization. The spectra are scaled with respect to the feature located at an energy loss of $325 \mathrm{eV}$. The Mo $\mathrm{M}_{4,5}$ edges show up as a broad delayed edge located at $225-325 \mathrm{eV}$. The two edges showing a white-line shape at $400 \mathrm{eV}$ are assigned to the Mo $\mathrm{M}_{2,3}$ edges. The presence of vanadium in the samples is evident from the $\mathrm{V} \mathrm{L}_{2,3}$ edges located at 517 and $524 \mathrm{eV}$ with the white-line nature in the preedge region of the $\mathrm{OK}$-edge threshold present at $532 \mathrm{eV}$. The apparent signals of the $\mathrm{V} \mathrm{L}_{2,3}$ edges are significantly more intense compared to the Mo $\mathrm{M}$ edges and the $\mathrm{O} \mathrm{K}$ edge in subset $\mathrm{B}$ catalysts. The analyzed areas in the subset $\mathrm{B}$ samples are more $\mathrm{V}$ rich than the samples from subset $\mathrm{A}$. The higher signals of the $\mathrm{O} \mathrm{K}$ edge in the spectra acquired from the subset B samples are due to the contribution from the higher vanadium signal. Quantitatively EDS analysis supports the variation in the $\mathrm{V}$ to Mo ratio in the two subsets. In the subset A samples the Mo: $\mathrm{V}: \mathrm{W}$ ratio is found to $70: 5: 25$, while the ratio in the subset $\mathrm{B}$ is $65: 12: 23$.

Fig. $9 \mathrm{~b}$ shows the electron loss near-edge structure of the $\mathrm{VL}_{2,3}$ edges and the $\mathrm{OK}$ edge. The $\mathrm{VL}_{3}$ edge and the $\mathrm{VL}_{2}$ edge are located at 516.9 and $523.8 \mathrm{eV}$, respectively. The $\mathrm{O} \mathrm{K}$-edge fine structure is dominated by a sharp threshold at $530.9 \mathrm{eV}$ and a broad feature located at $544 \mathrm{eV}$. The energy onset of the $\mathrm{VL}_{2,3}$ edges is identical in the spectra acquired from subset $\mathrm{A}$ and the subset $\mathrm{B}$ samples, although the latter are revealing more intense white lines due to the higher amount of vanadium. The $\mathrm{O} \mathrm{K}$ edge looks similar in the two cases.

\section{Discussion}

The results presented here emphasized the importance of characterizing the structure of catalyst material with methods more sensitive to defects or irregular structures as they might be of great importance in catalysis. Unfortunately, such methods are often more time consuming.

Chemical and elemental analysis by EELS is impossible of the very beam-sensitive structures as discussed above. The elemental composition of the amorphous material shown in Fig. $3 \mathrm{~b}$ is most likely different from the crystalline structure shown in Fig. 3a. However, Fig. 3a and all other acquired lattice images and their FFT of the beam-sensitive phases support a cubic structure with lattice parameter, $a \approx 2.2 \mathrm{~nm}$. The crystal in Fig. 3a is then oriented with the [011] zone axis parallel with the electron beam. This beam-sensitive phase might be related to the general high catalytic performance of the studied samples, but as this phase is observed in both subset A and subset B samples, the differences in the catalytic performance of the two subsets are presumably not to be found in the beam-sensitive phase. 
The crystalline structure of the catalyst samples studied in this work is predominated by the $\mathrm{Mo}_{5} \mathrm{O}_{14}$ type, with no or a very small amount of the fully oxidized orthorhombic $\mathrm{MoO}_{3}$ type, as no sign of this structure is found in the HRTEM images or electron diffraction patterns. Stoichiometric $\mathrm{MoO}_{3}$ is previously found to be inactive in acrolein oxidation. However, oxygen defects in this structure lead to a $\mathrm{MoO}_{3-x}$ catalyst favoring total oxidation [6 and 18]. Hence, orthorhombic $\mathrm{MoO}_{3-x}$ structures in the catalysts decrease the average selectivity for partial oxidation of propene.

The mixed metal oxides are homogeneously distributed in the agglomerate with no significant phase separation according to the elemental maps. The higher vanadium content in the subset B samples observed in quantitative EDS analysis and EELS must be considered as homogeneously distributed in the samples on a scale resolvable with EDS mapping.

The ELNES is known to reflect the local electronic structure and is highly sensitive to the local environment [ 30 and 31]. The coincidence of the energy loss of the $\mathrm{V} \mathrm{L}_{2,3}$ edges and the shape of the white lines in the spectra acquired from subset $\mathrm{A}$ and subset $\mathrm{B}$ samples indicate that the chemical environment of the additional vanadium atoms in the subset B samples is identical to the vanadium atoms present in the subset $\mathrm{A}$ samples. From the intensity ratio between the $\mathrm{V} \mathrm{L}_{3}$ and the $\mathrm{V} \mathrm{L}_{2}$ white line the oxidation state of the vanadium is estimated to be mainly $\mathrm{V}^{\mathrm{IV}}$. However, this estimate might be uncertain as the fingerprint spectra used for comparison of the white line ratio are acquired from pure vanadium oxides [32]. The O-K ELNES is superimposed by the fine structure from oxygen atoms bound to molybdenum, vanadium, and tungsten, with the sharp threshold at $530.9 \mathrm{eV}$ assigned mainly to the Mo-bonded oxygen. The higher amount of vanadium-bound oxygen at the expense of Mo- and $\mathrm{W}$-bound oxygen may cause the small difference in intensity found at $540 \mathrm{eV}$ in the two spectra in Fig. 9.

The structurally ill-defined phase observed at the surface of the core structure of the $\mathrm{Mo}_{5} \mathrm{O}_{14}$ type in the TEM images could be highly relevant for the understanding of the catalytic performance of the present catalysts in light of the mechanisms discussed above. The structurally ill-defined phase may serve as the prominent phase for adsorption of the organic substrate under selective $\mathrm{H}$ abstraction and oxygen insertion due to a high number of active sites. Previous studies have shown the same illdefined structure encapsulating a $\mathrm{Mo}_{5} \mathrm{O}_{14}$-type structure in thermally and acrolein-activated MoVW catalysts [16, 17 and 19]. The core of $\mathrm{Mo}_{5} \mathrm{O}_{14}$-type structure is suggested to act as an oxygen buffer due to its open structure of four-, six-, and sevenfold coordinated metal ions which allows oxygen diffusion to the surface as shown by XPS and RBS [16].

The origin to the difference in the catalytic performance of subset A and subset B samples may not be found in the subnanometer regime as the $\mathrm{Mo}_{5} \mathrm{O}_{14}$-type structure is observed in all the samples. The optimization of the crystal size must be addressed considering both the area of the ill-defined surface structure and the size of the $\mathrm{Mo}_{5} \mathrm{O}_{14}$-type core. If the core is too small the reaction kinetics might be 
limited by the reoxidation speed and the oxygen diffusion velocity. Hence, the optimum crystal size is the optimum ratio between structurally ill-defined surface and core (buffer) size. The difference in the morphology between the two subsets of catalysts studied in this work is reflected in the catalytic performance. The rough and porous surface of the subset A samples leads to a higher surface area of these samples. Furthermore, the crystal size observed in subset A catalysts might be closer to an optimum size, as the catalytic performance is better. However, the difference in the vanadium content in the two subsets might play a more direct role in the catalytic performance than the indirect role as a geometric promoter of MoVW-mixed oxides with $\mathrm{P}$ and $\mathrm{Cs}$ as additives.

HRTEM images of the high-performance selective partial oxidation catalysts support the model of structural well-defined crystals containing the motif of the $\mathrm{Mo}_{5} \mathrm{O}_{14}$-type structure, and more ill-defined surface structures of the crystals. The absence of orthorhombic $\mathrm{MoO}_{3}$ structures in the samples and optimization of the crystal size highly increase the performance of the catalyst. The determination and understanding of the catalytic active phase will lead to a more distinct path toward better selective partial oxidation catalysts.

\section{Conclusion}

Characterization of high-performance selective oxidation catalyst based on mixed oxides of molybdenum, vanadium, and tungsten by electron microscopy and associated techniques reveals a high amount of substoichiometric oxides. The general high performance of the catalyst studied in this work is assigned to the absence of orthorhombic $\mathrm{MoO}_{3}$ and the defective structure of the $\mathrm{Mo}_{5} \mathrm{O}_{14}$ type observed in the samples. Furthermore, a structurally ill-defined phase is found at the surface of the $\mathrm{Mo}_{5} \mathrm{O}_{14}$-type cores. These types of structures highly relevant for the catalytic reactions cannot be fully revealed by bulk-sensitive techniques, such as XRD. The difference in the catalytic performance of the studied samples is to be found in the morphology of the samples rather than in the crystallographic structure.

Defective structures such as the present $\mathrm{Mo}_{5} \mathrm{O}_{14}$-type structure and the structurally ill-defined layer found on the surface of some of the crystals in the samples are not revealed in the corresponding XRD results, emphasizing the importance of using nonintegral techniques when studying structures fundamental in heterogeneous catalysis, such as surfaces. 


\section{References}

1. R.K. Graselli. Catal. Today 49 (1999), p. 141.

2. P. Mars and D.W. Krevelen. Chem. Eng. Sci. 3 (1954), p. 41.

3. K. Brückman, R. Grabowski, J. Haber, A. Maurkiewicz, J. Slocynski and T. Wiltowski. J. Catal. 104 (1987), p. 71.

4. A.B. Anderson, D.W. Ewing, Y. Kim, R.K. Graselli, J.D. Durrington and J.F. Brazdil. J. Catal. 96 (1985), p. 222.

5. A.R. Adams and T.J. Jennings. J. Catal. 3 (1964), p. 549.

6. J. Haber and E. Lalik. Catal. Today 33 (1997), p. 119.

7. A. Tenten, F.-G. Martin, H. Hibst, L. Marosi, V. Kohl, BASF AG, EP 668108 B1, 1995

8. T. Kawajiri, S. Uchida, H. Hironaka, Nippon Shokubai Kagaku, EP 427508 A1, 1991

9. V. Novak, L. Sokol, J. Jelinik, CS 1207807 B, 1981

10. N. Bertolini, S. Ferlazzo, US patent 4289654, 1981

11. A.N. Kurtz, R.W. Cunningdam, A.W. Naumann, US patent 4111983, 1978

12. S. Breiter, M. Estenfelder, H.-G. Lintz, A. Tenten and H. Hibst. Appl. Catal. A 134 (1996), p. 81.

13. K. Krauss, A. Drochner, M. Fehlings, J. Kunert and H. Vogel. J. Mol. Catal. A 162 (2000), p. 413.

14. L.B. Levy and P.B. Groot. J. Catal. 76 (1982), p. 385.

15. P.B. Groot and L.B. Levy. J. Catal. 76 (1982), p. 393.

16. G. Mestl, C. Linsmeier, R. Gottschall, M. Dieterle, J. Find, D. Herein, J. Jäger, Y. Uchida and R. Schlögl. J. Mol. Catal. A 162 (2000), p. 455.

17. O. Ovsitser, Y. Uchida, G. Mestl, G. Weinberg, A. Blume, M. Dieterle, H. Hibst and R. Schlögl. J. Mol. Catal. A 185 (2002), p. 291.

18. M. Dieterle, PhD thesis, TU Berlin, 2001

19. M. Dieterle, G. Mestl, J. Jäger, Y. Uchida, H. Hibst and R. Schlögl. J. Mol. Catal. A 174 (2001), p. 169.

20. G. Mestl. J. Raman Spectrosc. 33 (2002), p. 333.

21. H. Werner, O. Timpe, D. Herein, Y. Uchida, N. Pfänder, U. Wild, R. Schlögl and H. Hibst. Catal. Lett. 44 (1997), p. 153.

22. L. Kihlborg. Ark. Kemi 21 (1963), p. 427.

23. T. Ekström and M. Nygren. Acta Chem. Scand. 26 (1972), p. 1827.

24. T. Ekström and M. Nygren. Acta Chem. Scand. 26 (1972), p. 1836.

Preprint of the Department of Inorganic Chemistry, Fritz-Haber-Institute of the MPG (for personal use only) (www.fhi-berlin.mpg.de/ac) 
25. T.V. Andrushkev and T.G. Kuznetsova. Kinet. Katal. 27 (1986), p. 571.

26. L.M. Plyasova, L.P. Solovéva, G.N. Kryukova and T.V. Andruschkevich. Kinet. Katal. 31 (1989), p. 1253.

27. T.V. Andrushkev. Catal. Rev.-Sci. Eng. 35 (1993), p. 213.

28. R.K. Graselli and J.D. Burrington. Adv. Catal. 30 (1981), p. 133.

29. J.D. Burrington, C.T. Kartisek and R.K. Graselli. J. Catal. 87 (1984), p. 363.

30. V.J. Keast, A.J. Scott, R. Brydson, D.B. Williams and J. Bruley. J. Microsc. 203 (2001), p. 135.

31. R.F. Egerton, Electron Energy—Loss Spectroscopy in the Electron Microscope., Plenum, New York (1996).

32. M. Willinger, Diploma thesis, Vienna University of Technology, 2001 


\section{Figures}
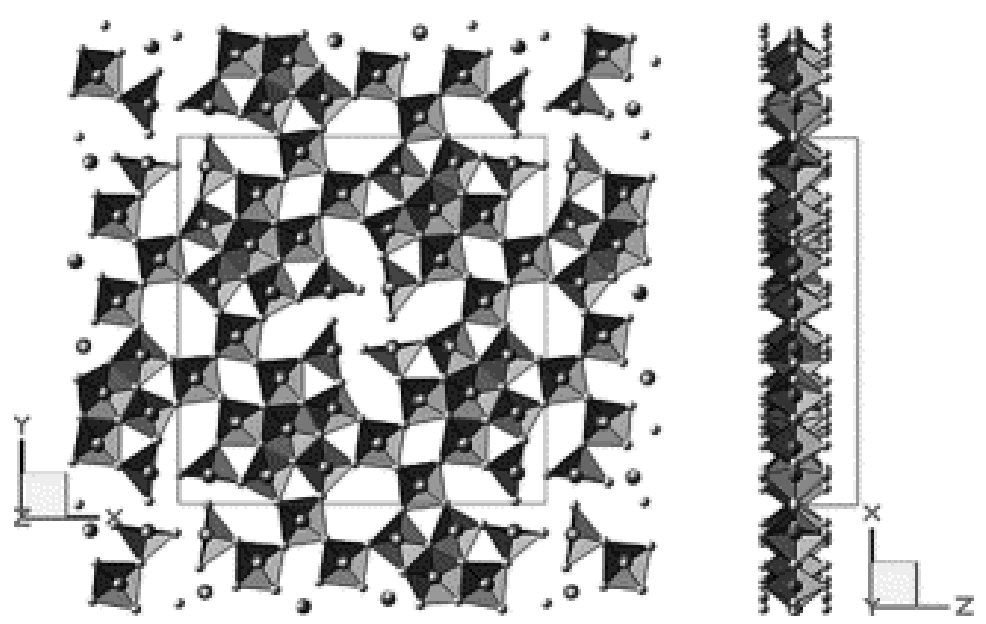

Fig. 1. Schematic drawing of the $\mathrm{Mo}_{5} \mathrm{O}_{14}$ structure. The structure is shown along the [001] zone axis (left) and the [100] zone axis (right). The unit cell is outlined in both drawings. The building blocks consist of pentagonal bipyramids and octahedrally coordinated metal centers leading to a twodimensional open structure.
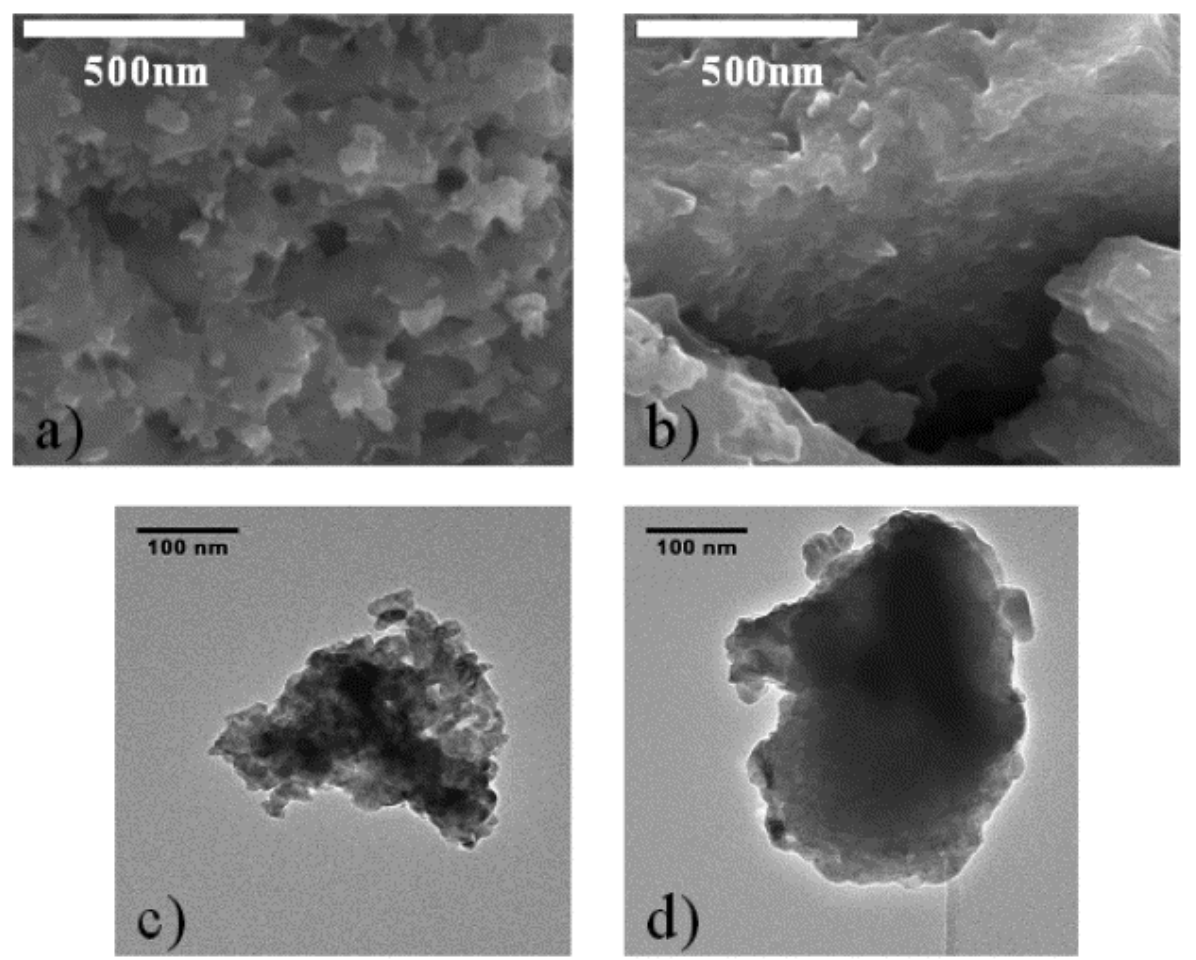

Fig. 2. SEM images and corresponding low-magnification TEM images of (a) and (c), a subset A sample, and (b) and (d), a subset B sample. 


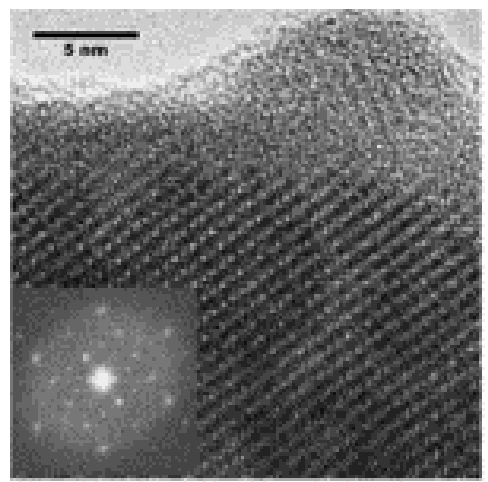

(a)

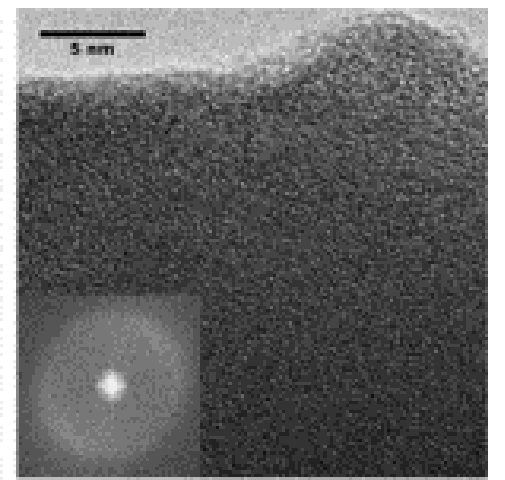

(b)

Fig. 3. (a) High-resolution TEM image of the highly beam-sensitive crystalline phase. (b) Same area as in (a) after less than 1 min of electron irradiation. The phase has become amorphous. The insets show the FFT of the corresponding images.

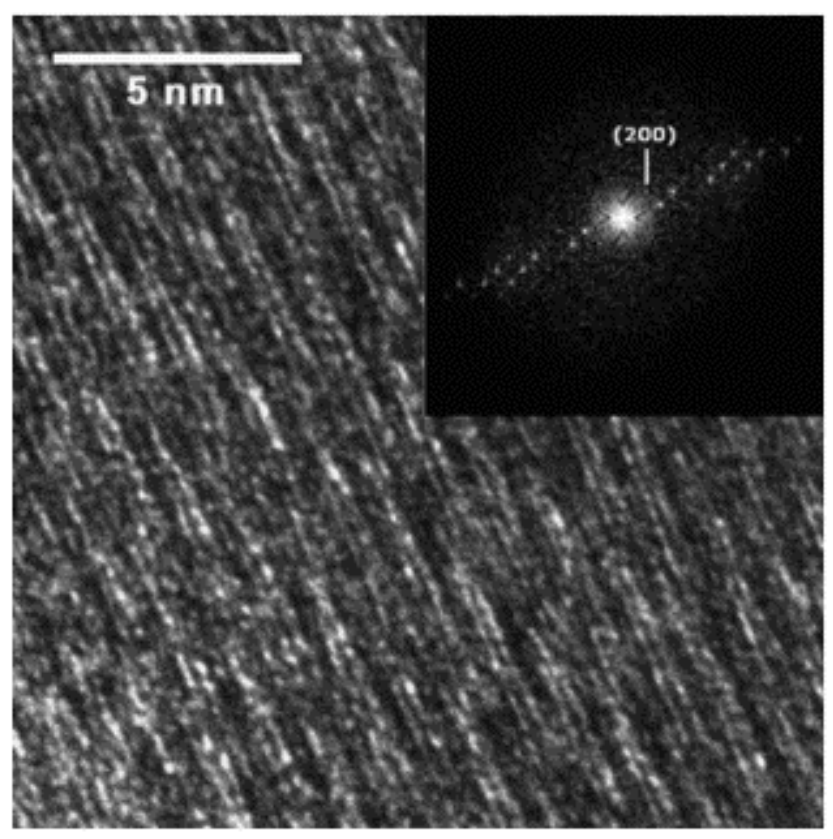

Fig. 4. Lattice fringe image of $\mathrm{Mo}_{5} \mathrm{O}_{14}$-type structure slightly tilted from the [001] zone axis. The FFT of the image reveals spots spaced with a distance corresponding to $2.25 \mathrm{~nm}$ in real space. 


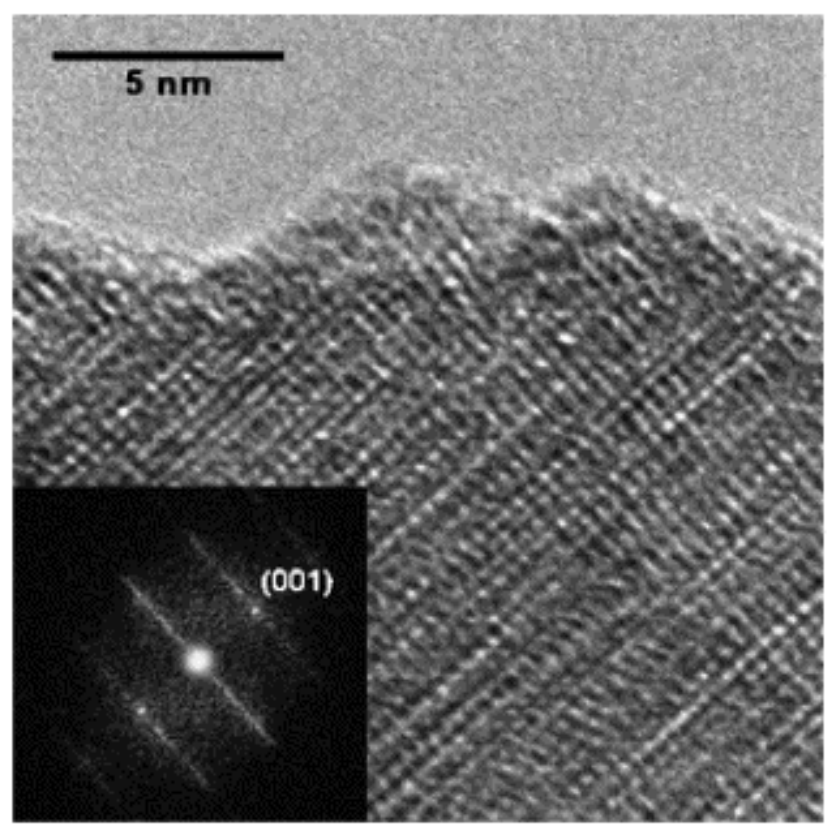

Fig. 5. Pseudolamellar structure in the $\mathrm{Mo}_{5} \mathrm{O}_{14}$-type structure. The FFT of the HRTEM image reveals streaks indicating the large dimensions of the unit cell.

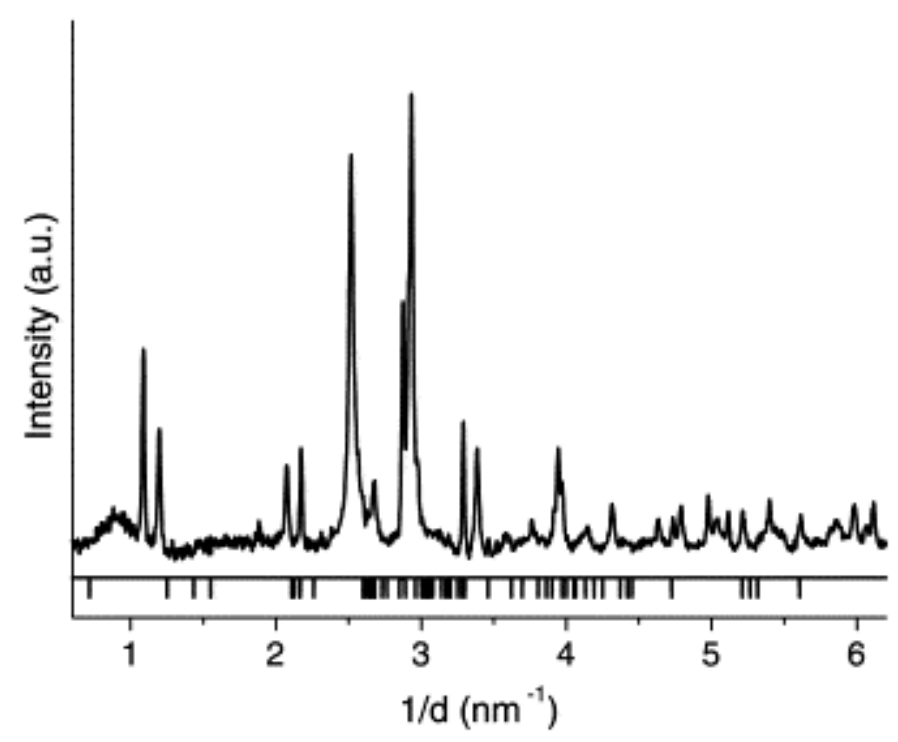

Fig. 6. Typical XRD pattern of the catalyst samples compared with lattice spacings found in the HRTEM. The XRD patterns do not reveal the defective structures in the samples. 

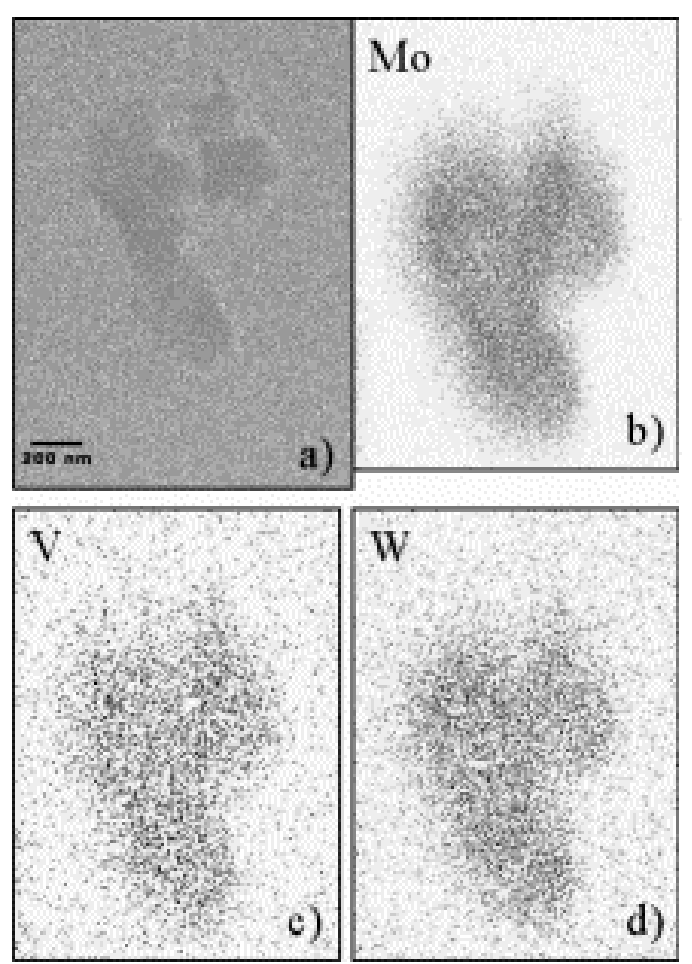

Fig. 7. Elemental map of Mo, V, and $\mathrm{W}$ of a sample from subset A acquired by EDAX in STEM mode. The metals are equally distributed throughout the agglomerates.

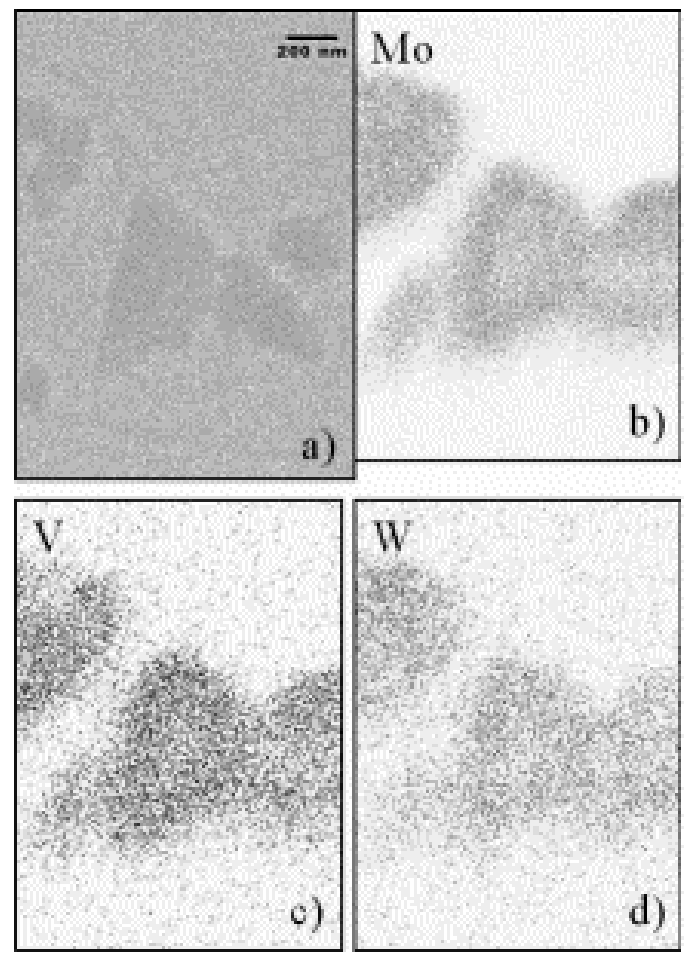

Fig. 8. Elemental map of Mo, $\mathrm{V}$, and $\mathrm{W}$ of a sample from subset $\mathrm{B}$ acquired by EDAX in STEM mode. The metals are equally distributed throughout the agglomerates. 


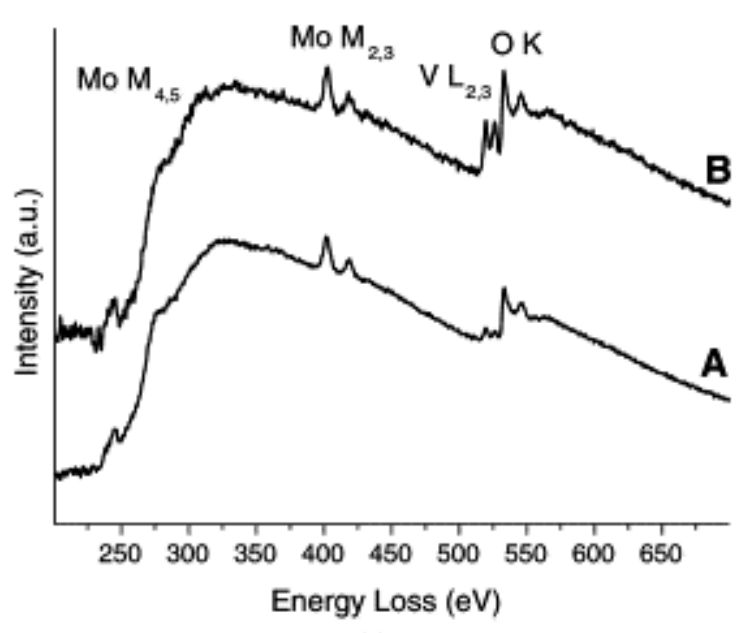

(a)

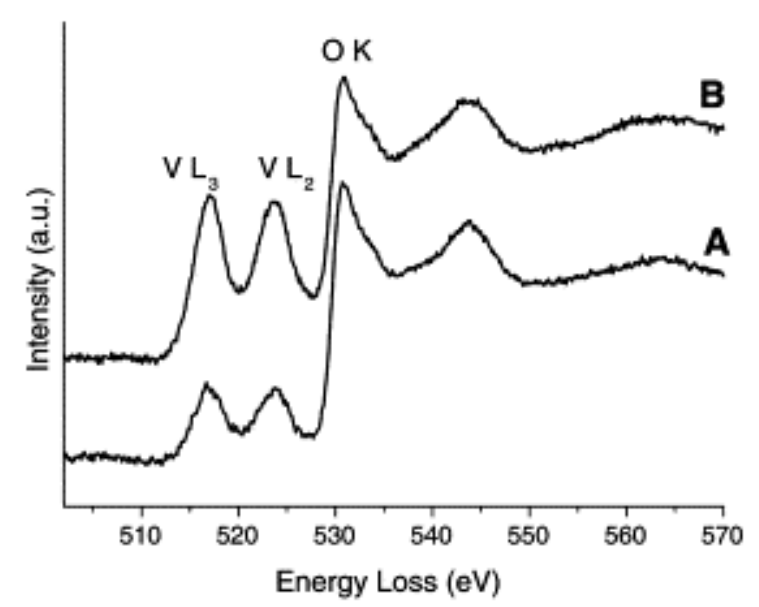

(b)

Fig. 9. Electron-loss spectra of samples from subset A and subset B. (a) The Mo M edges, the $\mathrm{V} L$ edges, and the $\mathrm{O} \mathrm{K}$ edge are present in the spectra. The vanadium content is seen to be highest in subset B samples. (b) ELNES of the V L edges and the O K edge. 